\title{
Neutron anisotropic evaporation and scission emission in fission
}

\author{
L. Stuttgé ${ }^{1, *}$, A. Chietera ${ }^{1, \dagger}$, F. Gönnenwein ${ }^{2}$, Yu. Kopatch ${ }^{3}$, M. Mutterer ${ }^{4,5}$, A. Gagarski ${ }^{6}$, \\ I. Guseva ${ }^{6}$, E. Chernysheva ${ }^{7}$, F.-J. Hambsch ${ }^{8}$, F. Hanappe ${ }^{9}$, Z. Mezentseva ${ }^{3}$, \\ S. Telezhnikov ${ }^{3}$ \\ ${ }^{1}$ Université de Strasbourg, CNRS, IPHC UMR 7178, F-67000 Strasbourg, France \\ ${ }^{2}$ Physikalisches Institut, Universität Tübingen, D-72076 Tübingen, Germany \\ ${ }^{3}$ Frank Laboratory of Neutron Physics, JINR, 141980 Dubna, Russia \\ ${ }^{4}$ GSI Helmholtzzentrum für Schwerionenforschung, D-64291 Darmstadt, Germany \\ ${ }^{5}$ Institut für Kernphysik, Technische Universität Darmstadt, D-64289 Darmstadt, Germany \\ ${ }^{6}$ Petersburg Nuclear Physics Institute, National Research Centre "Kurchatov Institute", \\ 188300 Gatchina, Russia \\ ${ }^{7}$ Flerov Laboratory of Nuclear Reactions, JINR, 141980 Dubna, Russia \\ ${ }^{8}$ EC-JRC-Institute for Reference Materials and Measurements, B-2440 Geel, Belgium \\ ${ }^{9}$ PNTPM, Université Libre de Bruxelles, B-1050 Brussels, Belgium
}

\begin{abstract}
Experimental neutron distributions have been investigated in the spontaneous fission of ${ }^{252} \mathrm{Cf}$ at IPHC in Strasbourg. The CORA experiment associating the CODIS twin ionisation chamber and the neutron multi-detector DEMON aimed to solve an long-standing problem in fission: the possible emission of scission neutrons and/or the presence of a dynamical anisotropy in the neutron evaporation by the moving fission fragments. A new method allowing to establish the dynamical anisotropy in an independent way is presented. The results obtained from a comparison with simulations based on GEANT4 are shown.
\end{abstract}

\section{Introduction}

It is commonly considered that during the fission process the major part of the neutrons are emitted isotropically by the fully accelerated fragments. But many works, experimental and theoretical, showed up discrepancies with such a purely isotropic emission. The question arises to find out the origin of this deviation, either due to some dynamical anisotropy in the centres of mass $(\mathrm{CM})$ of the fission fragments $(\mathrm{FF})$ or to neutron emission at scission.

Already in 1962, H. Bowman et al. [1] made a detailed measurement of the neutrons emitted in spontaneous fission of ${ }^{252} \mathrm{Cf}$. In the evaluation the authors had to introduce $10 \%$ of scission emission to explain their results. The possibility of some anisotropy in the CMs of the fragments was also suggested. B. Franklyn et al. [2], when they analysed K. Skarsvåg and K. Bergheim's data on thermal neutron induced fission of ${ }^{235} \mathrm{U}$, had to assume up to $20 \%$ scission emission to reproduce the measured distributions. Even with

\footnotetext{
* Corresponding author: stuttge@in2p3.fr

${ }^{\dagger}$ Present address: Irfu, CEA, Université Paris-Saclay, F-91191 Gif-sur-Yvette, France
} 
this huge contribution of scission emission there were still discrepancies. More recently, theoretical calculations [3] investigated the influence of anisotropy in the CMs of the FFs on the angular distribution. The effect appears to be very weak. It slightly reinforces the kinematic focusing in the laboratory system. Moreover, as shown in figure 1, scission neutron emission and dynamical anisotropy act on the angular correlation in an opposite way.
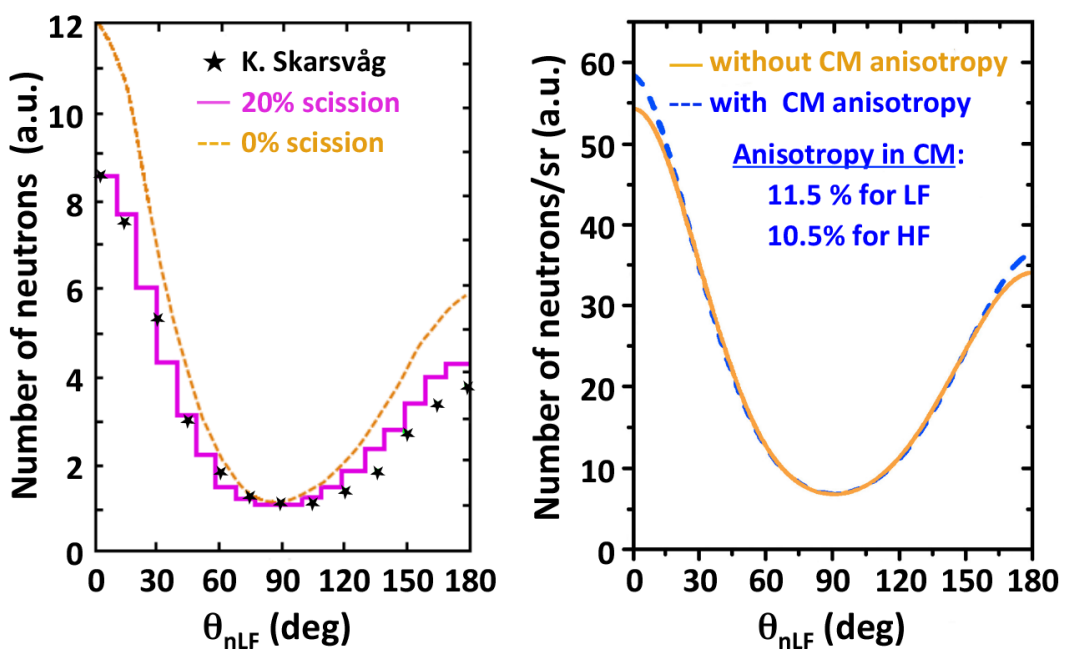

Fig. 1. Neutron angular distributions as a function of the angle between the emitted neutrons and the light fragment (LF) in the thermal neutron induced fission of ${ }^{235} \mathrm{U}$ :

- left: scission neutrons taken into account. Adapted from [2]

- right: dynamical anisotropy taken into account. Adapted from [3].

Much work has addressed this problem of ambiguity but with contradictory results. Usually people restricted their study to one of the mentioned processes but not simultaneously to both. The angular configuration of the DEMON detector used in the CORA experiment allowed to approach dynamical anisotropy and scission neutron emission, simultaneously in the same measurement.

\section{New method to extract the dynamical anisotropy}

An important innovation brought up by F. Gönnenwein [4,5] and implemented in the CORA experiment is a new method to extract the dynamical anisotropy in the CMs of the FFs, in an independent way from any theoretical model and in which neither the kinematic focusing nor the scission emission interfere. Triple or higher coincidences between any FF and at least two associated neutrons have to be considered. In case of an extreme anisotropy, which can be imagined due to the high spin values of the emitting FFs, all neutrons will be emitted in a plane perpendicular to the spin of the emitting FF. When projected onto a plane perpendicular to the fission axis, these neutron events will be located on a single line, the $\mathrm{x}$-axis as shown in figure 2 .

This situation has to be compared to the case of perfect isotropy where the neutrons are randomly spread over the (x,y) plane as sketched in figure 2 which shows the case of a particular orientation of the spin. In experiment all spin orientations around the fission axis are measured and the neutron lines will follow the spin orientations. The azimuthal angular difference between any two neutrons will thus be $0^{\circ}, 180^{\circ}$ or $360^{\circ}$ in case of extreme 
anisotropy, depending on the sign of the $\mathrm{x}$-coordinate. This distribution has to be compared to the flat distribution obtained for the perfect isotropy as shown on the bottom of figure 2 .

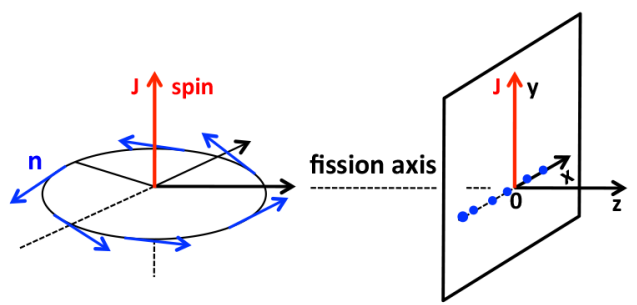

extreme $\mathrm{CM}$ anisotropy

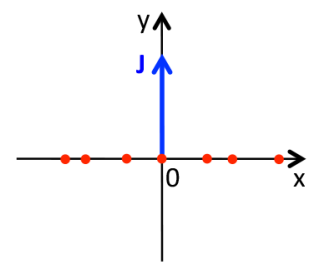

all n-events on a line
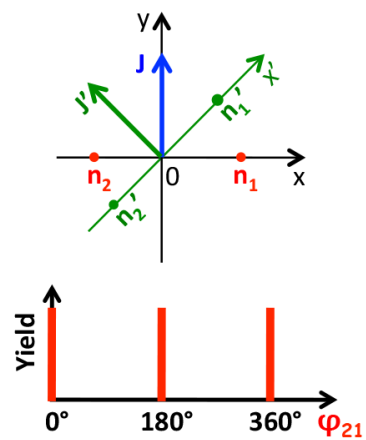

perfect CM isotropy

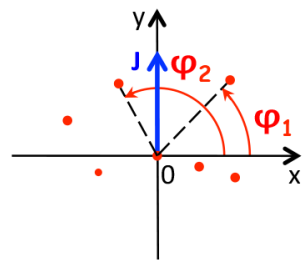

n-events distributed isotropically in plane

$$
\varphi_{21}=\varphi_{2}-\varphi_{1}
$$

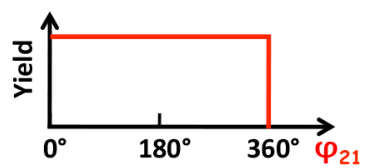

Fig. 2. Extreme anisotropy in the CM of the FF: all neutrons are emitted in a plane perpendicular to the spin axis (top). Projection plane $x-y$ : the projection lines follow the inclination of the spin orientations compared to the random distribution in the case of perfect isotropy. Azimuthal angle difference distributions, $\varphi_{21}$, in both extreme cases (bottom). The real case is in between these two extreme cases.

The actual distribution is expected to be in between these two extremes and can be expressed according to I. Guseva [6] as

$$
W\left(\varphi_{n n F}\right) \propto 1+a_{2} \cos ^{2} \varphi_{n n F}
$$

where $\varphi_{n n F}$ is the azimuthal angle difference between any two neutrons as presented in figure 2 and the $a_{2}$ parameter represents the magnitude of dynamical anisotropy, also expressed as the anisotropy ratio

$$
a_{2}=\frac{W\left(0^{\circ}\right)-W\left(90^{\circ}\right)}{W\left(90^{\circ}\right)}
$$

This method allows to disentangle the dynamical anisotropy component from the kinematic focusing due to the transformation from the CM to the laboratory system. It will also be practically free from any influence of a possible scission neutron emission. 


\section{CORA experiment}

The setup for the CORA experiment was composed of the angle-sensitive twin ionization chamber CODIS [7] for the detection of fission fragments and sixty modules of the DEMON [8] neutron multi-detector as shown in figure 3.

Fig. 3. CORA setup:

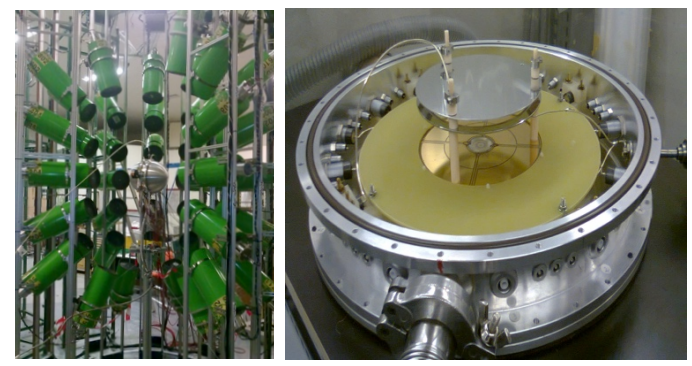

- left: 60 DEMON cells are arranged in a near spherical configuration around the CODIS fission chamber

- right: view of the open CODIS chamber with the ${ }^{252} \mathrm{Cf}$ source in the centre of the segmented cathode.

CODIS provides the energies, mass ratios and emission angles of the FFs from the central ${ }^{252} \mathrm{Cf}$ source while DEMON gives the energies and angles of the correlated neutrons. Sixty DEMON modules have been used in the actual CORA setup.

In a five-month experiment, about $10^{9}$ triple or higher coincidences between any FF and two or more associated neutrons have been collected.

The geometrical configuration used for DEMON, almost spherical around the CODIS chamber, allowed to study for the first time both processes, dynamical anisotropy and scission neutron emission, simultaneously in the same experiment.

\section{CORA analysis}

For the many parameters measured in the CORA experiment several experimental biases concerning particularly the neutron detection had to be considered carefully. The geometrical acceptance of DEMON was about $20 \%$ of $4 \pi$. A common neutron energy threshold was set at $0.9 \mathrm{MeV}$. The intrinsic efficiency of the DEMON modules and the fact that only the centres of the DEMON cells can be used to define the detection geometry had to be taken into account. Another important effect is the cross talk, when one emitted neutron interacts successively with two detectors, mainly in neighbouring detectors. In the actual geometry, the cross talk is quite low but, as the effect of interest is also very weak, it may impact the result.

Detailed simulations based on GEANT4 have been performed in order to consider the experimental biases but also to get a feeling about the extracted quantities and to check the relevance of the analysis.

Three correlations were addressed: $\varphi_{n n F}$ as defined above, the neutron-neutron relative angles $\theta_{n n}$ and the angles between a light fragment (LF) and the neutrons, $\theta_{n L F}$.

The implementation of the simulations are described in detail in references $[9,10]$ and preliminary results were presented in $[11,12]$. The characteristics of the FFs (mean velocities, mean temperatures) and the neutrons (multiplicities, variances, covariance) were taken from literature $[13,14,15]$. As to the neutrons, the angles have been generated randomly for an anisotropic emission in the CMs of the FFs. The dynamical anisotropy has 
been introduced by generating a $\cos \theta_{C M}$ distribution following the prescription of I. Guseva [6]:

$$
W\left(\theta_{C M}\right) \propto 1+A_{n J} \sin ^{2} \theta_{C M}
$$

where $A_{n J}$ is the anisotropy parameter with respect to the spin of the FF and is related to the $a_{2}$ parameter of eq. 2 as shown in figure 4 . The neutron energies are taken in a Maxwellian distribution.

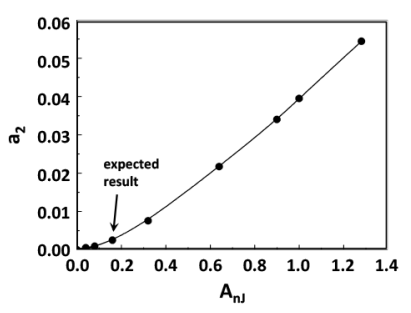

Fig. 4. Anisotropy ratio $a_{2}$ as a function of the anisotropy parameter $A_{n J}$ [6]. The line links the calculated values.

To add scission neutrons, an isotropic emission in the laboratory system was assumed and the scission neutron energies were taken from a Weisskopf evaporation distribution.

For illustration figure 5 shows the simulation result for the $\theta_{n n}$ distribution, starting from an isotropic emission (top-left) and taking into account the experimental filter (geometrical acceptance, energy threshold, intrinsic efficiency, cross talk, etc.). The resulting distribution (top-right) can be compared with the experimental one (bottom-right).
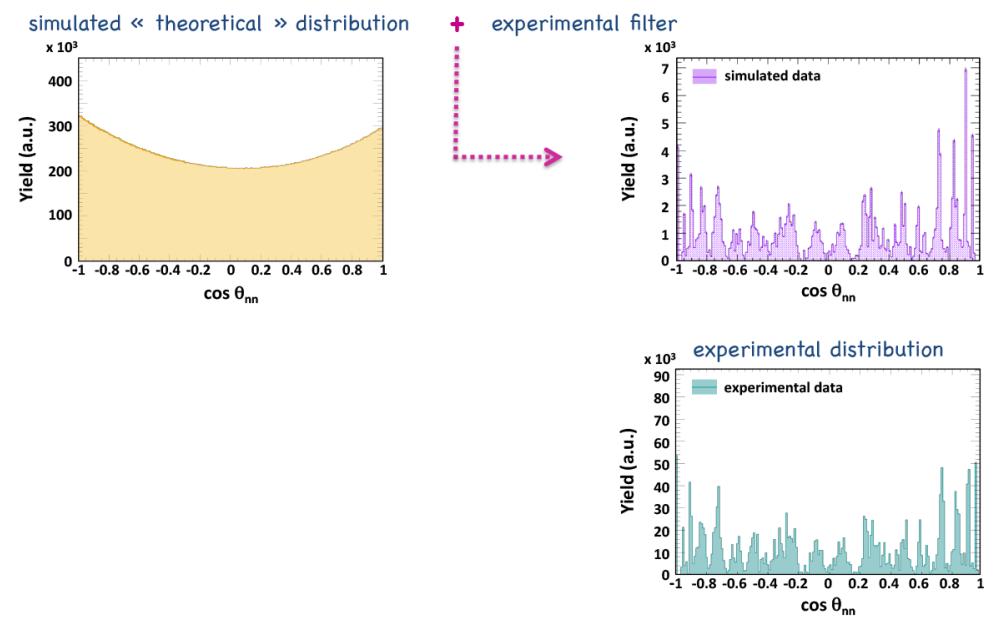

Fig. 5. Comparison of the simulated $\theta_{n n}$ distribution (top-right), resulting from an isotropic neutron emission (top-left) when the experimental filter is applied, with the experimental distribution (bottomright).

At first glance, the two distributions closely agree and only the small discrepancies appearing around $\cos \theta_{n n}= \pm 1$ are the indicator of a possible scission emission and/or a dynamical anisotropy contribution. In order to obtain a valuable comparison, both distributions have to be corrected for the response function of the detector which, due to its geometry, introduces pronounced narrow structures in the angles. 
This response function was determined by considering uncorrelated events both in the simulated and in the experimental distributions [10].

\section{Results}

Figure 6 presents the resulting correlations $\varphi_{n n F}$ (left), $\theta_{n n}$ (right-top) and $\theta_{n L F}$ (rightbottom).

The $\varphi_{n n F}$ distribution has been fitted following eq. 1 . The fit result is shown in figure 6 (red curve). The deduced anisotropy ratio $a_{2}=0.0028 \pm 0.0015$ is consistent with I. Guseva's prediction, $a_{2}=0.0025$ as indicated in figure 4 , corresponding to an anisotropy parameter $A_{n J}=0.16$. However the large reduced $\chi^{2}$ value of the fit shows that beyond the statistical error there is a systematic error which has been estimated. This leads to a total error estimated to be of the order of $4 \times 10^{-3}$.

This value was then fixed in the simulation to extract the scission emission component by comparing the experimental $\theta_{n n}$ and $\theta_{n L F}$ distributions (blue points) to the simulated ones (pink curves) by a $\chi^{2}$ minimisation. The best fit is obtained for a scission emission probability $\omega_{s c i}=8 \%$ which is also in agreement with the result of [15].

Only the angles $\theta_{n n}>53^{\circ}$ are presented in figure 6 . This restriction, applied also to the $\varphi_{n n F}$ distribution, was necessary to remove the impact of cross talk events to the analysis.
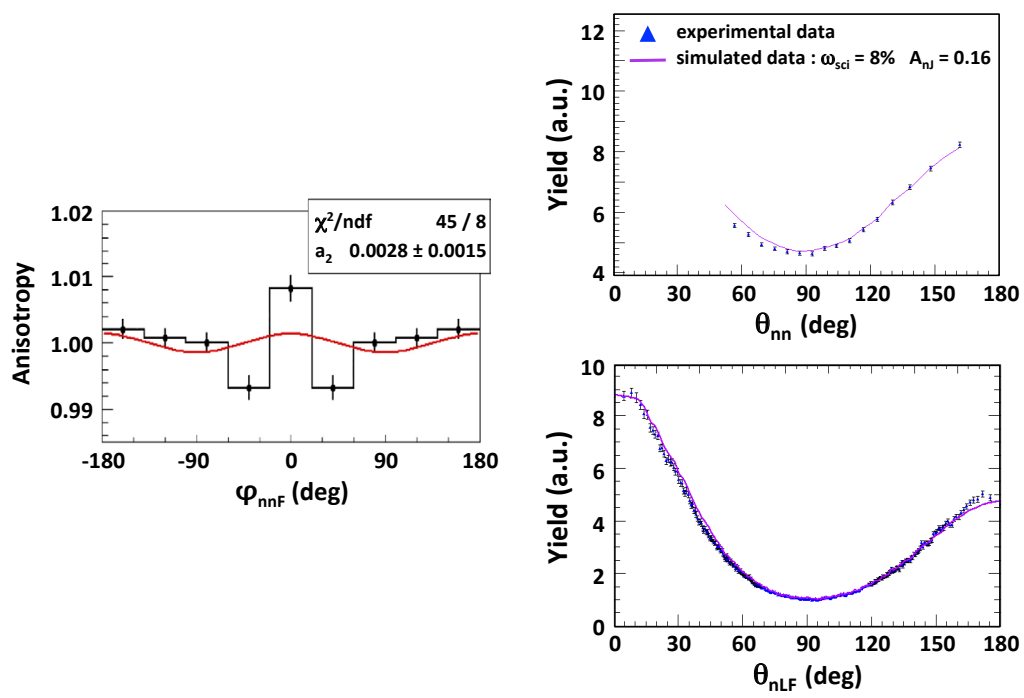

Fig. 6. Summary of the experimental results after the three distributions $\varphi_{n n F}, \theta_{n n}$ and $\theta_{n L F}$ have been corrected for the detector response by normalisation to uncorrelated events:

- left: The resulting distribution has been fitted by eq. 1 . The fit parameters are presented in the insert.

- $\quad$ right: The normalised $\theta_{n n}$ (top) and $\theta_{n L F}$ (bottom) experimental distributions (blue points) have been compared to the corresponding simulated ones (pink curves) by a $\chi^{2}$ minimisation leading to a scission emission probability $\omega_{s c i}=8 \%$ for both distributions. The agreement between experiment and simulation is very good.

The slightly high threshold at $0.9 \mathrm{MeV}$ of the measured neutron energies shouldn't influence the present results as its effect has been taken into account in the simulations. Concerning the anisotropy, according to reference [3], its effect increases drastically with neutron energy. Thus the major part of those neutrons have been detected in the CORA 
experiment. As for the scission neutrons, their distribution is not really known up to now. In our simulation a temperature of Tsci $=1.2 \mathrm{MeV}$ adopted from [15] has been used in the Weisskopf formula. If this approach is realistic, also a reduced amount of those scission neutrons only have been missed in the experiment.

\section{Conclusion}

The CORA experiment constitutes a new approach to address the problem of ambiguities in the interpretation of the fission neutron angular distributions. CORA which associated CODIS and DEMON is the first experiment giving access simultaneously to both the dynamical anisotropy and the scission neutron emission.

It allowed to accede to the contributions of these two processes quantitatively. The values obtained up to now, $A_{n J}=0.16$ and $\omega_{s c i}=8 \%$, are consistent with theoretical calculations performed by I. Guseva.

Complementary work is needed to confirm the value obtained for the $A_{n J}$ anisotropy parameter as its contribution is very weak and the error attached to the $a_{2}$ determination is still quite high. However, the present work demonstrates the relevance of the new approach.

This work was partially supported by the JINR-IN2P3 agreement for which the authors are very grateful.

\section{References}

1. H. R. Bowman et al., Phys. Rev. 126, 2120 (1962)

2. C. B. Franklyn et al., Phys. Lett. B 78, 564 (1978)

3. V. E. Bunakov, I. S. Guseva et al., Proc. Int. Seminar "ISINN-13", Dubna, Russia, World Scientific, 175 (2006)

4. F. Gönnenwein et al., Proc. "Seminar on Fission", Corsendonk Priory, Belgium, World Scientific, 3 (2008)

5. L. Stuttgé et al., Proc. "Seminar on Fission", Het Pand, Gent, Belgium, World Scientific, 181 (2011)

6. I. S. Guseva, Proc. Int. Seminar "ISINN-23", Dubna, Russia, World Scientific, 80 (2016)

7. Yu. N. Kopatch et al., Phys. Rev. C 65, 044614 (2002)

8. I. Tilquin et al., Nucl. Instr. Methods A 365, 466 (1995)

9. A. Chietera et al., submitted to EPJA

10. A. Chietera, $\mathrm{PhD}$ thesis, Université de Strasbourg (2015), Unistra $\mathrm{n}^{\circ} 3064$

11. A. Chietera et al., Acta Polonica B 46, 569 (2015) and Proc. Int. Seminar "ISINN-22", Dubna, Russia, World Scientific, (2015)

12. Y. Kopatch et al., Proc. "Scientific Workshop on Nuclear Fission dynamics and the Emission of Prompt Neutrons and Gamma Rays, THEORY-3", Opatija, Croatia, Physics Procedia 64, 171 (2015) and "Int. conf. on nuclear data for science and technology", ND2016, Bruges, Belgium

13. N. V. Kornilov et al., Nucl. Phys. A 686, 187 (2001)

14. N. V. Kornilov et al., INDC(CCP)-435, 61 (2002)

15. A. M. Gagarski, I. S. Guseva et al., Abstract Int. Seminar "ISINN-20", Dubna, Russia, $42(2013)$ 